GENERAL SURVEILLANCE

\title{
The National Violent Death Reporting System: an exciting new tool for public health surveillance
}

\author{
M Steenkamp, L Frazier, N Lipskiy, M DeBerry, S Thomas, L Barker, D Karch
}

See end of article for

authors' affiliations

....................

Correspondence to:

Dr L Barker, Centers for

Disease Control and

Prevention, National

Center for Injury

Prevention and Control,

4770 Buford Highway,

$\mathrm{NE}$, Mailstop K-60,

Atlanta, GA, 30341 USA;

Isb8@cdc.gov

Accepted 31 July 2006
Injury Prevention 2006;12(Suppl II):ii3-ii5. doi: 10.1136/ip.2006.012518

\begin{abstract}
The US does not have a unified system for surveillance of violent deaths. This report describes the National Violent Death Reporting System (NVDRS), a system for collecting data on all violent deaths (homicides, suicides, accidental firearms deaths, deaths of undetermined intent, and deaths from legal intervention, excluding legal executions) in participating states. The NVDRS centralizes data from many sources, providing a more comprehensive picture of violent deaths than would otherwise be available. The NVDRS collects data on victims, suspects, and circumstances related to the violent deaths. Currently, 17 US states participate in the NVDRS; the intention is for the NVDRS to become a truly national system, representing all 50 states, the District of Columbia, and the US territories. This report describes the history of the NVDRS, provides an overview of how the NVDRS functions, and describes future directions.
\end{abstract}

$\mathrm{V}$ iolent deaths from homicide and suicide took more than 49000 lives in the US in 2003. ${ }^{1}$ Homicide and suicide are, respectively, the third and fourth leading causes of death for people aged 1-39 years. ${ }^{2}$ Despite this, only basic national data are available on these violent deaths; there is no national surveillance system for violence in the US and many of the existing national data sources do not provide data that are sufficiently detailed to inform policy and program decisions. ${ }^{3}$ As a result, rhetoric and anecdote, rather than evidence, drives much of the public debate about preventing violence and the consequences of violence.

National reporting systems that track the incidence and characteristics of health conditions have been well established in areas such as infectious disease and motor vehicle safety. These systems have provided necessary data to drive prevention efforts. A national system for surveillance of violent deaths in the US could, similarly, provide knowledge needed to prevent violent deaths. Routinely collected data on violent deaths could also aid in evaluating strategies and programs for the prevention of violent deaths. The development of a uniform violent death reporting system across state and local areas would facilitate evaluation by providing both historical and cross sectional data useful for assessing the impact of strategies and programs.

In 1999, six private foundations pooled their resources to begin the National Violent Injury Statistics System (NVISS), a national fatal intentional injury surveillance system that included 12 pilot sites. The NVISS developed many of the tools and methods necessary for multisource surveillance of violent deaths. In 2000, the Harvard Injury Control Research Center and the Joyce Foundation convened a meeting of experts that suggested that the Centers for Disease Control and Prevention (CDC) direct a publicly funded system similar to NVISS. ${ }^{3}$

In 2002, Congress appropriated $\$ 1.5$ million to begin development and implementation of a population based, active surveillance system. ${ }^{3}$ This system, called the National Violent Death Reporting System (NVDRS), was designed to provide a census of resident and occurrent violent deaths that occur within the US. The NVDRS is an active state-based surveillance system. Its ultimate purpose is to provide accurate, timely, and comprehensive surveillance data that will allow us to reduce and prevent the occurrence of violent deaths in the US.

\section{ADVANTAGES OF THE NVDRS}

The NVDRS requires no additional data collection; all data are collected by other existing data systems. However, the NVDRS makes a unique contribution by providing a centralized data repository. It is impossible to characterize the problems of homicide and suicide in sufficient detail with current national data sources, since they exist in isolation. For example: national mortality data compiled from death certificates filed in the states lack sufficient information about such factors as the circumstances of the event, type and characteristics of the weapon, characteristics of the offender, type of location where the violent death occurred, and indications of drug or alcohol involvement; data on crimes collected by the Federal Bureau of Investigation in the Uniform Crime Reporting System are also limited with respect to some of the above variables and do not include suicides; data from medical examiners' and coroners' offices are not collected in any central location; and important national injury surveillance systems, such as the Consumer Product Safety Commission's National Electronic Injury Surveillance System, do not collect data on fatal injuries. In contrast, the NVDRS can answer many questions that these systems, due to dispersal of data and lack of details, cannot (for example, rate of occurrence of linked homicide/suicide suicide, defined as an incident in which the suspect commits suicide within 24 hours of committing homicide; percent of homicides in which a legally intoxicated victim was killed by a blunt instrument; percent of youth suicides motivated by legal troubles which occur within the victim's home).

\section{GROWTH}

The NVDRS data collection began in 2003, with six states (Maryland (MD), Massachusetts (MA), New Jersey (NJ), Oregon (OR), South Carolina (SC), and Virginia (VA)). Since

Abbreviations: CDC, Centers for Disease Control and Prevention; ICD10, International Classification of Diseases, 10th revision; NVDRS,

National Violent Death Reporting System; NVISS, National Violent Injury Statistics System. 


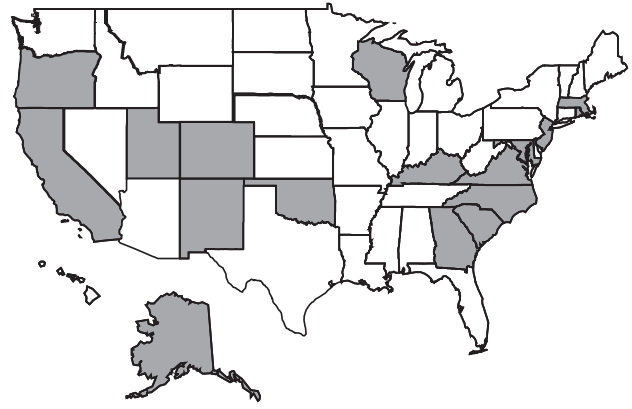

Figure 1 States participating in the National Violent Death Reporting System as of May 2006.

then, two additional appropriations, in 2003 and 2004, have allowed 11 additional states to be funded, bringing the total to 17 (fig 1). Alaska (AK), Colorado (CO), Georgia (GA), North Carolina (NC), Oklahoma (OK), Rhode Island (RI), and Wisconsin (WI) began collecting data for 2004. One state (AK) funded in 2004 additionally collected 2003 data retrospectively. California (CA), Kentucky (KY), New Mexico (NM), and Utah (UT) began collecting data for 2005. The seven states collecting 2003 data combined accounted for $12.5 \%$ of the US population in 2003 . The 13 states participating in 2004 accounted for $23.2 \%$ of the 2004 US population. The goal is to eventually fund all 50 states, the District of Columbia, and the US territories.

\section{Scope of the NVDRS data collection}

For the NVDRS purposes, a violent death is defined as a death that results from the intentional use of any means to injure or poison oneself, another person, or group of people. ${ }^{4}$ A firearm related death is defined as one that results from projectile injuries from a gun that uses an explosive charge for a propellant. The NVDRS collects information on all violent deaths and accidental firearms related deaths in participating states. To assess misclassification, the NVDRS also includes deaths of unknown intent. The NVDRS therefore includes all suicides, homicides, deaths of undetermined intent, deaths resulting from legal intervention (excluding legal executions), and deaths from unintentional firearm injuries occurring in the funded states. The system excludes deaths due to acts of war, but includes deaths due to terrorism related events.

The NVDRS collects information on victims (deceased persons) and alleged perpetrators (deceased or live suspects). Data on deaths belonging to the same incident (for example, a linked homicide/suicide or multiple homicide) are tracked

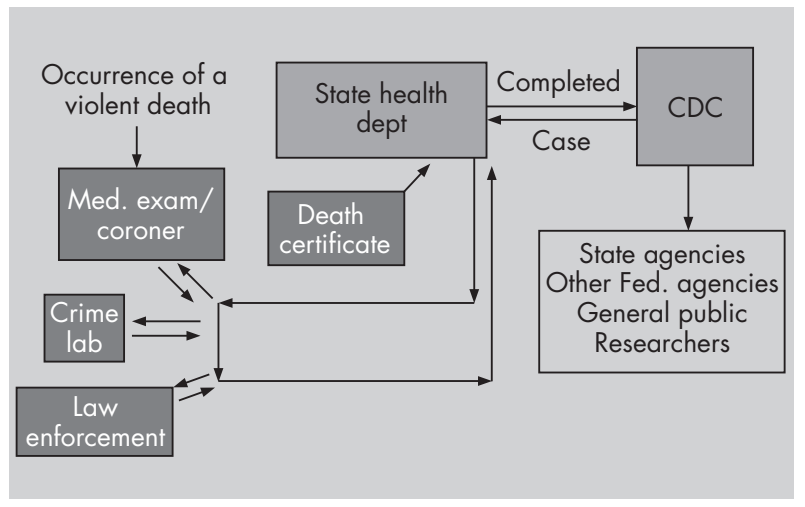

Figure 2 Flow of data within the National Violent Death Reporting System. together. Accordingly, data within the NVDRS can be analyzed on the victim or suspect level (for example, to determine details about victims or suspects) or the incident level (in which multiple homicides or homicides/suicides can be considered as single events).

Details are collected on a large number of sociodemographic characteristics of deceased persons and basic demographic characteristics of both alive and deceased suspects. The system collects additional information on circumstances contributing to the deaths, such as (for suicides and deaths of undetermined intent) circumstances related to mental health, disclosed intent, and precipitating factors; and felony related or non-felony related circumstances for homicides. In addition, the NVDRS captures data on interpersonal relationships (for example, the suspect was the victim's spouse), victim toxicology (for example, the victim's blood alcohol content), and the mechanisms of injury (for example, blunt instrument, poisoning, etc).

State health departments or their bona fide agents serve as the lead organizations for the projects and the centralized points where data from law enforcement, medical examiners/ coroners, vital records, and crime laboratories are collected, linked, and stored. Cases are typically identified either when the death certificate is filed or when an abstractor reviews cases reported to coroner/medical examiner (CME) offices. Each state's own Violent Death Reporting System establishes the details of that state's cases from primary and secondary data sources. Primary data sources are: death certificates (DC); CME records; police reports (PR); and crime laboratory data. Secondary or optional data sources are: child fatality review team data (CFR); supplementary homicide reports (SHR); hospital (Hosp) data; emergency department (ED) data; and Alcohol, Tobacco, Firearms and Explosives (ATF) trace information on firearms.

Because the NVDRS is state based, states can choose to capture data beyond what the CDC collects. States can also choose to collect data that law enforcement and other agencies that have a role in violence prevention deem valuable. Thus, states can analyze data that are richer than those available to the CDC. This capacity means that additional data collected by each state will and should look different.

\section{Data coding and analyses}

Data from multiple sources (for example, DC, CME records, police reports) are entered independently into distinct fields in the database. Some of the more than 740 data elements are the same data entered from multiple sources, because these sources might disagree-for example, the system collects manner of death from three sources (the DC, CME records, and the data abstractor) and demographic details from four sources (the DC, CME records, SHR, and PR). A primacy rule, which assigns to each data source an order of authoritativeness, is used to create CDC analysis variables. For example for sex, primacy states that the DC is considered the most authoritative source, followed by, in order, the CME, SHR, and PR. If sex is missing from the DC field, the analysis variable takes sex from the CME field if that is available. If that is not available, the value is taken from the SHR field, etc. Data are taken from all the different data sources as available. In all states, trained abstractors assign manners of death to all cases using standard NVDRS definitions after reviewing information from all available sources.

All participating states are provided CDC-developed data collection software, which automatically flags inconsistent data entry (for example, out-of-range data). Data are pushed nightly from the state to a vendor for processing. The CDC provides feedback to states regarding the quality of their data (for example, inconsistencies between narrative description of the deaths and the variables checked). States are 
encouraged to develop their own data quality protocols, in addition to those the CDC provides. The states, in turn, provide data quality feedback and reports to the sources from which they received the data (fig 2).

Data are added to the system as they become available, typically on a monthly basis. The time lag for the various data sources varies from two months (death certificate data) to two years or more (law enforcement data). In some states, law enforcement does not release data regarding a homicide until the case has been adjudicated, resulting in potentially very long lags. The NVDRS is still in the process of evaluating the level of missing data after a given time period.

Data presented in this supplement come mainly from DCs, CME data, and law enforcement records. All papers reflect data for 2003 and 2004, either singly or together, and represent data as it stood in July 2005; 2003 and 2004 data are subject to possible further revision, which might refine, but is unlikely to overturn, findings of reports in this supplement. For all rate calculations, we used the National Center for Health Statistics-provided intercensal and postcensal bridged race population estimates. Rates were age adjusted to the year 2000 national standard population. The abstractor-assigned manner of death was used for all analyses in this supplement. International Classification of Diseases, 10th revision (ICD-10) codes for the underlying cause of deaths were not used because ICD-10 codes have, as of this writing, not yet been reported to the NVDRS for 150 (1\%) and 2078 (15\%) cases for 2003 and 2004, respectively.

\section{Future directions for the NVDRS}

The NVDRS opens a new chapter in the use of scientific information to guide public policy and prevention efforts concerning violence in the US. Even though it is not yet a truly national system, the NVDRS provides valuable insight into violent deaths through surveillance on a large number of characteristics of violent deaths, including circumstances. Further, as the NVDRS develops, it can serve as a model for other nations that want to develop surveillance systems for violent deaths within their borders.

This supplement is just one of the strategies that will be used to disseminate NVDRS data. The CDC is planning to create an automated data query system similar to the Webbased Injury Statistics Query and Reporting System (WISQARS). Such a system would allow researchers and the general public alike to quickly and easily obtain violent death reports and data.

\section{ACKNOWLEDGEMENTS}

A national reporting system of this kind requires the contributions of many agencies and organizations to facilitate data collection

\section{Key points}

- The National Violent Death Reporting system collects data on violent deaths (Suicides, homicides, deaths of undetermined intent, deaths by legal intervention, and accidental firearms deaths) in participating states of the US.

- The system coordinates data from death certificates, coroner/medical examiner reports, police reports, and a variety of other sources that would otherwise not be linked.

- Although the system requires no additional data collection, its multisource approach provides a more comprehensive view of violent deaths in the US than would otherwise be available.

and guide the system's development and implementation. We acknowledge the vital contributions of the Violent Death Reporting Systems of the states of AK, CA, CO, GA, KY, MD, MA, NJ, NM, NC, OK, OR, RI, SC, VA, UT, and WI. We also acknowledge the data providers to the Violent Death Reporting Systems in each funded state-that is, state health departments, vital registrars' offices, coroners' and medical examiners' offices, crime laboratories, local and state law enforcement agencies. Finally, we want to thank the reviewers, whose comments allowed us to strengthen this manuscript.

\section{Authors' affiliations}

M Steenkamp, L Frazier, N Lipskiy, M DeBerry, S Thomas, L Barker, D Karch, Centers for Disease Control, National Center for Injury Prevention and Control, Atlanta, GA, USA

The findings and conclusions in this report are those of the authors and do not necessarily represent the views of the Centers for Disease Control and Prevention.

\section{REFERENCES}

1 Centers for Disease Control and Prevention, National Center for Injury Prevention and Control. Web-Based Injury Statistics Query and Reporting System (WISQARS), 2005. Available at http://www.cdc.gov/ncipc/wisqars (accessed May 2006).

2 NCHS Vital Statistics System for numbers of deaths, Bureau of Census for population estimates. Statistics compiled by the Office of Statistics and Programming, NCIPC, CDC

3 Paulozzi L, Mercy J, Frazier L, et al. CDC's National Violent Death Reporting System: background and methodology. Inj Prev 2004;10:47-52.

4 Azrael D, Barber C, Mercy J. Linking data to save lives: recent progress in establishing a National Violent Death Reporting System. Harvard Health Policy Review $2001 ; 2: 38-42$. 